\title{
Fingertip Dosing Unit
}

National Cancer Institute

\section{Source}

National Cancer Institute. Fingertip Dosing Unit. NCI Thesaurus. Code C71321.

An arbitrary dosing unit used predominantly for semisolid topical formulations such as cream, ointment, paste, etc. One fingertip unit is the amount of a product that is squeezed out from a standard tube with 5-millimeter diameter nozzle along an adult's fingertip. A fing ertip length is defined from the tip of the index finger to the first finger crease. A fing ertip dosing unit varies with age and size of the body. The average fingertip unit is equal to approximately 0.5 gram for an adult male and 0.4 gram for an adult female. 